\title{
Aperiodically poled structures for high efficiency broadband terahertz generation
}

\author{
Koustuban Ravi $^{1,2}$, Alireza Yahaghi ${ }^{1}$, Arya Fallahi ${ }^{1}$, and Franz X.Kärtner ${ }^{1-3}$ \\ 1. Center for Free-Electron laser Science, DESY, Notkestraße 85, Hamburg 22607, Germany \\ 2. Research Laboratory of Electronics, Massachusetts Institute of Technology, 50 Vassar Street, Cambridge MA 02139. \\ 3. Department of Physics and The Hamburg Center of Ultrafast Imaging, University of Hamburg, Hamburg 22761, Germany. \\ koust@mit.edu
}

\begin{abstract}
We introduce a combination of aperiodically poled structures and chirped mirrors for broadband terahertz generation. Unprecedented spectral and temporal shaping possibilities with energy conversion efficiencies $>5 \%$ and terahertz output energies of $\sim 10 \mathrm{~mJ}$ are predicted.

OCIS codes: (190.0190) Nonlinear optics; (300.6495) Terahertz spectroscopy
\end{abstract}

\section{Introduction}

Single or few-cycle broadband terahertz radiation with large peak electric fields offer unique possibilities in fundamental investigations of light-matter interaction [1]. Additionally, the unique combination of long wavelengths and large peak field strengths has garnered interest in technological applications such as electron acceleration and beam diagnostics [3]. In particular, single-cycle pulses with energies significantly larger than the milli-joule level offer the possibility of accelerating electrons from rest to very high energies on the order of $\mathrm{MeV}$ over very short length scales. Such high gradient acceleration can lead to unprecedented electron beam properties which might be transformative for coherent X-ray generation, electron microscopy and medical therapy. However, the largest broadband pulse energies reported to date are $0.9 \mathrm{~mJ}$ [4] and the path to scaling single-cycle pulse energies to the 10 $\mathrm{mJ}$ level is currently unclear.

The tilted-pulse-front technique pioneered in [5] is able to produce conversion efficiencies $>1 \%$ and can leverage on the powerful $1 \mu \mathrm{m} / 800 \mathrm{~nm}$ laser technology. However, scaling to very large pump energies or conversion efficiencies is limited by angular dispersion related issues [6]. As a solution, organic materials [4] possessing very high nonlinearity and semiconductor based approaches with significantly smaller angular dispersion [7] have been proposed. However, the latter class of approaches relies on the development of lasers with wavelengths $>1.3 \mu \mathrm{m}$, which are significantly less developed compared to $1 \mu \mathrm{m}$ laser technology.

Here we introduce a new set of approaches based on aperiodically poled crystals which achieve phase-matching in a collinear geometry, thereby circumventing the angular dispersion limitations of tilted-pulse-fronts. However, they may still be pumped by the highly developed $1 \mu \mathrm{m}$ laser technology. The generated chirped terahertz pulses are subsequently compressed by chirped mirrors designed for the terahertz wavelength range. The collinear geometry allows for recycling of the optical pump in several stages, promising conversion efficiencies as large as $5 \%$ for terahertz radiation centered at $\sim 0.3 \mathrm{THz}$ in cryogenically cooled lithium niobate crystals. Importantly, by customizing the aperiodic poling, one may shape both the terahertz spectrum and waveform. Using current state-ofthe-art poling technology with $\mathrm{cm}^{2}$ apertures [8], terahertz pulse energies at the $5 \mathrm{~mJ}$ level may be generated. With further increase in aperture sizes, this may be further extended beyond the $10 \mathrm{~mJ}$ level.

\section{Results}

Rigorous and experimentally verified numerical simulations [9] accounting for the coupled nonlinear interaction of optical and terahertz radiation were employed. The assumed material was lithium niobate with a bulk second order nonlinearity $\chi^{(2)}=336 \mathrm{pm} / \mathrm{V}$ and crystal temperature of $100 \mathrm{~K}$. The pump fluence was limited by laser induced damage and was assumed to scale with pulse duration as $F_{\text {pump }}=0.1(\tau / 1 \mathrm{ps})^{0.5} \mathrm{Jcm}^{-2}$. The basic mechanism of terahertz generaton in an aperiodically poled crystal is depicted in Fig.1a. An optical pump pulse propagates through the crystal while generating a broadband terahertz pulse. The optical pulse propagates faster in lithium niobate and consequently, additional cycles are added to the front of the initially generated cycles. Since absorption is larger for larger terahertz frequencies, the poling period is gradually reduced to minimize absorption loss. Naturally, the terahertz pulse thus generated is chirped. In Fig.1b, we depict the resultant terahertz spectrum for a pump pulse of duration $\tau=800 \mathrm{fs}$, and crystal length of $\mathrm{L}=1 \mathrm{~cm}$. The optimal poling profile was generated using hill-climbing optimization routines. Subsequently, the terahertz pulses may be compressed using a chirped terahertz mirror 
composed of alternating layers of quartz and air, backed by a metallic layer as shown in Fig.1c. Using nonlinear optimization techniques, a chirped mirror design which compresses the pulses to a few cycles is shown in Fig.1c.

(a)

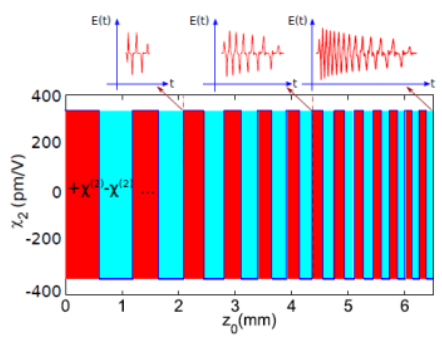

(b)



(c)



Fig.1(a). Mechanism of terahertz generation in an aperiodically poled crystal producing chirped terahertz pulses. (b) Broadband terahertz spectrum for a crystal optimized for performance in the frequency range $<1 \mathrm{THz}$. (c) Chirped terahertz mirrors composed of alternating quartz and air layers, backed by copper to compress the chirped terahertz pulses from (a).

Upon passage through a single crystal, conversion efficiencies of approximately $2 \%$ are obtained. However, due to the absence of angular dispersion limitations, the pump may be recycled in subsequent stages yielding conversion efficiencies as high as $5 \%$ in aggregate as depicted in Fig.2. Upon passage through each stage, the optical pump spectrum undergoes increasingly larger red-shift due to cascading. With current $\mathrm{cm}^{2}$ apertures, energies as large as 5 $\mathrm{mJ}$ may be obtained with readily available $100 \mathrm{~mJ}$ pump pulses. However, with further increase in aperture areas, pump energies can be increased offering the possibility of surpassing the $10 \mathrm{~mJ}$ limit.

stage 1
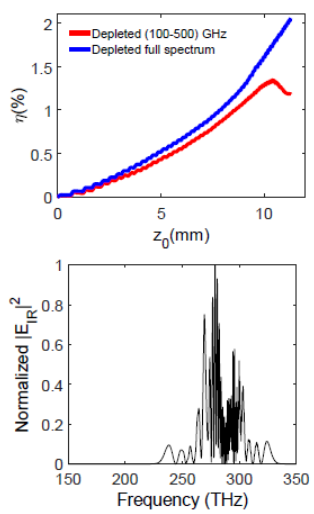

stage 2
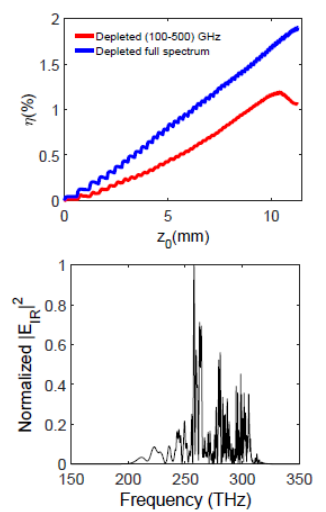

stage 3
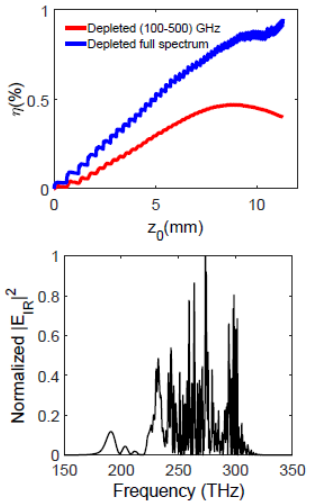

stage 4
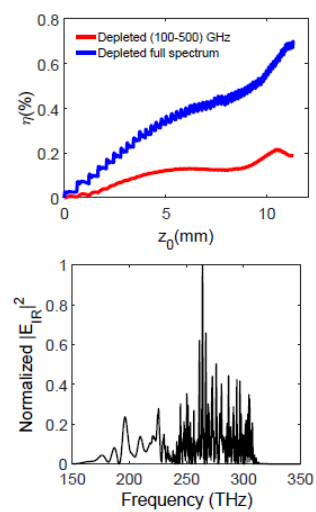

Fig.2. The collinear geometry of aperiodically poled crystals circumvents angular dispersion limitations and allows for pump recycling. Cumulative conversion efficiencies $>5 \%$ are deemed possible using such structures. The optical pump spectrum shows continuous red-shift at each stage due to cascading.

\section{Conclusion}

In summary, a different approach to terahertz generation using aperiodically poled crystals and chirped mirrors was introduced to address the limitations of existing generation methods. The approach offers very large conversion efficiencies in a collinear geometry with possibilities of spectral and temporal shaping of the pulses as well as pump recycling. Using large area poled crystals, the approach can be foreseen as one way to breach the next frontier in high energy single-cycle terahertz generation.

\section{References}

[1] O.Schubert, M.Hohenleutner, F.Langer, B.Urbanek, C.Lange, U.Huttner, D.Golde, T.Meier, M.Kira, S.W.Koch and R.Huber, Nature Photonics 8, 119-123 (2014).

[2]W.R.Huang, A.Fallahi, X.Wu, H.Cankaya, A.L.Calendron, K.Ravi, D.Zhang, E.A.Nanni, K.H.Hong, and F.X.Kärtner, “Terahertz-driven alloptical electron gun, " Optica 3,1209-1212 (2016).

[3]C.Kealhofer, W.Schneider, D.Ehberger, A.Ryabov, F.Krausz, and P.Baum, Science 352, 429-433 (2016).

[4] C. Vicario, A. Ovchinnikov, S. Ashitkov, M. Agranat, V. Fortov, and C. Hauri Optics letters 39, 6632 (2014).

[5] J. Hebling, G. Almasi, I. Kozma, and J. Kuhl, Optics Express 10, 1161 (2002).

[6] K. Ravi et al., "Limitations to THz generation by optical rectification using tilted pulse fronts," Opt. Express 22, 20239 (2014).

[7] J. Fülöp, G. Polónyi, B. Monoszlai, G. Andriukaitis, T. Balciunas, A. Pugzlys, G. Arthur, A. Baltuska, and J. Hebling, Optica 3, 1075 (2016).

[8] T. Taira and H. Ishizuki, Opt. Express 22(16), 19668-19673(2014).

[9] K.Ravi , D.N.Schimpf, and F.X.Kärtner, Optics Express 24, 25582 (2016). 